EPJ Web of Conferences 100, 03002 (2015)

DOI: $10.1051 /$ epjconf/201510003002

(C) Owned by the authors, published by EDP Sciences, 2015

\title{
Gamma radiation exposure of accompanying persons due to $\mathrm{Lu}-177$ patients
}

\author{
Bilal Kovan ${ }^{1, a}$, Bayram Demir², Duygu Tuncman², Veli Capali ${ }^{2}$, Cuneyt Turkmen ${ }^{1}$ \\ ${ }^{1}$ Istanbul University, Istanbul Medical Faculty, Nuclear Medicine Department, 34093, Istanbul, Turkey \\ ${ }^{2}$ Istanbul University, Science Faculty, Physics Department, 34134, Istanbul, Turkey \\ ${ }^{3}$ Süleyman Demirel University, Arts and Sciences Faculty, 32260, Isparta, Turkey
}

\begin{abstract}
Neuroendocrine tumours (NET) are cancers usually observed and arisen in the stomach, intestine, pancreas and breathing system. Recently, radionuclide therapy applications with Lu-177 peptide compound are rapidly growing; especially effective clinical results are obtained in the treatment of well-differentiated and metastatic NET. In this treatment, Lu-177-DOTA, a beta emitter radioisotope in the radiopharmaceutical form, is given to the patient by intravenous way. Lu-177 has also gamma rays apart from beta rays. Gamma rays have $175 \mathrm{keV}$ average energy and these gamma rays should be under the control in terms of radiation protection. In this study, we measured the exposure dose from the Lu-177 patient.
\end{abstract}

\section{Introduction}

Neuroendocrine tumors (NET) are cancers usually observed and arisen in the stomach, intestine, pancreas and breathing system. Recently, radionuclide therapy applications with $\mathrm{Lu}-177$ peptide compound are rapidly growing; especially effective clinical results are obtained in the treatment of well-differentiated and metastatic NET. In this treatment, Lu-177-DOTA, a beta emitter radioisotope in the radiopharmaceutical form, is given to the patient by intravenous way. This targeting radiopeptide is intensely accumulated in the tumor site containing somatostatin receptor and the tumor is internally treated by means of beta rays emitted from Lu177. The treatment is sequentially repeated 4 or 5 times in the 6-8 weekly periods [1].

Lu-177 is a beta emitter with a maximum energy of 0.5 $\mathrm{MeV}$ and it's a maximal tissue penetration of $2 \mathrm{~mm}$. Lu177 half-life is 6.7 days. A part from beta rays, Lu-177 has also emits two low-energy $\gamma$-rays at 208 and $113 \mathrm{keV}$ with $10 \%$ and $6 \%$ abundance. These gamma rays allow scintigraphy and subsequent dosimetry with the same therapeutic compound. Because of the gamma rays of the $\mathrm{Lu}-177$, radiation protection issue can be became a problem. We measure the dose exposure resulted from Lu-177 patient to accompanying person [2].

\section{Material and Method}

In this study, we measured the radiation exposure of four Lu-177 patients. Each patient was injected with average $200 \mathrm{mCi} \mathrm{Lu}-177$ with $500 \mathrm{cc}$ serum during 30 minutes.
After the injection, patient's stayed in an isolated room for the clinical follow-up during one day.

Radiation exposure measurements were performed by using a portable Ludlum trademark Geiger Muller device. Geiger Muller was calibrated by Turkish Atomic Energy Authority. After injection, measurements were performed at 4. hours, 24. hours, 48. hours, 96. hours and 120. hours. Measurement distances were 0.2, 0.5 and 1 meter from the patients' abdominal regions. Measurement results were given in Table 1.

\section{Results}

Neuroendocrine tumours (NETs) develop in the cells of the neuroendocrine system. There are several types of neuroendocrine tumors. These types are Gastrointestinal neuroendocrine tumours, Pulmonary neuroendocrine tumours and other NETs, known as functioning tumors. One of the treatment model of the NETs tumours is radiation therapy with radionuclides. Lu-177 is known to be effective in neuroendocrine tumours, paragangliomas, neuroblastomas and certain types of thyroid cancer. Lu177 is a radioactive substance that we can add to a carrier called DOTATATE. Once in your body, the Lu-177 DOTATATE attaches to specific tumour cells and destroys these cancerous cells. [3]

Lu-177 emits beta rays, besides gamma radiation. It has gamma rays which can be considered as dangerous in terms of radiation protection. Injected doses are $200 \mathrm{mCi}$ in the Lu-177 treatment. On the other hand, NETs are advanced cancer type and these patients need close patient care $[4,5]$. Therefore, these people are at risk of exposure to radiation. In this study, we measured the dose

a e-mail : bkovan@istanbul.edu.tr 
exposure resulted from Lu-177 patient to accompanying person. Therefore, measurement distance was selected $0.2,0.5$ and $1 \mathrm{~m}$. Especially $0.2 \mathrm{~m}$ measurements are important because of gamma rays energies of Lu-177. Its energies are 113 and $208 \mathrm{keV}$. These energies affects to the accompanying person in the case of close contact. Various values for six patients are shown in Figs. 1, 2 and 3. Significant degradation is observed from the first 24 hours for each distances $(0.2 \mathrm{~m}, 0.5 \mathrm{~m}$ and $1 \mathrm{~m})$ when the figures were analysed. After 24 hours, Lu-177 activity keeps in organs. Then, this activity decreases slowly. Thus first 24 hours very critical for accompanying persons. Because of radiation exposure for accompanying persons, they should refrain from close contact with their patients. Moreover patients should be kept in isolated rooms after the injection.

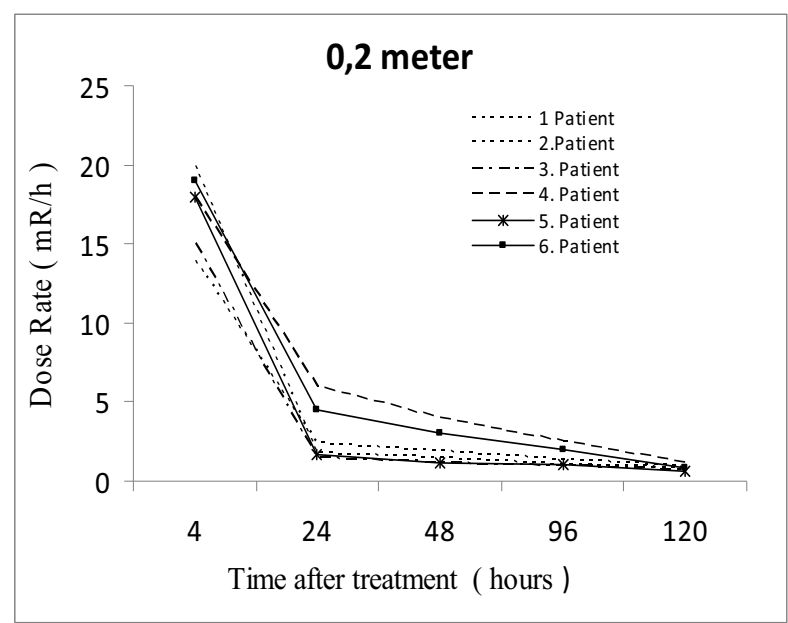

Figure 1. Dose rate measurements results at $0.2 \mathrm{~m}$.

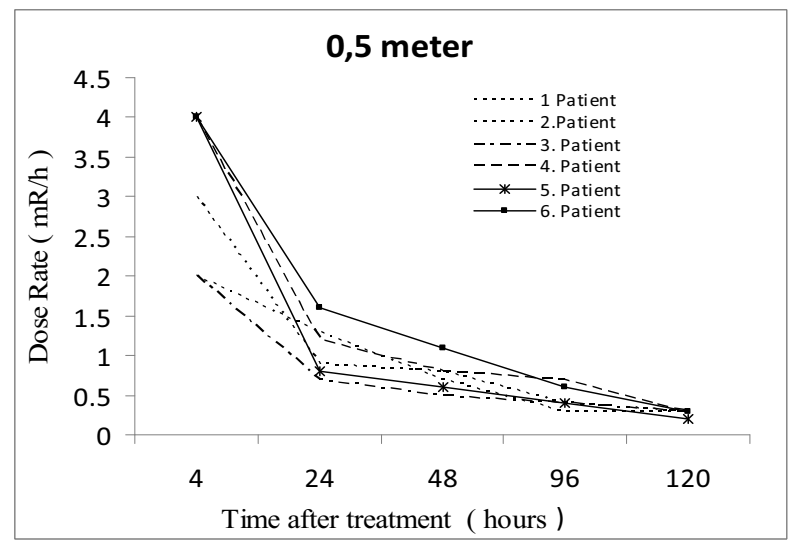

Figure 2. Dose rate measurements results at $0.5 \mathrm{~m}$.

\section{Conclusion}

Lu-177 treatment is sequentially repeated 4 or 5 times in the 6-8 weekly periods, each fraction has $200 \mathrm{mCi}$ activity. Totally $800-1000 \mathrm{mCi}$ Lu-177 activity was injected to the patient's body. Gamma rays energies of Lu-177 are considered as important. So it is important to keep in an isolated room for at least one day after the injection. First 24 hours close contact should be avoided for each distance from accompanying persons to patients. Periodical measurements should be made after Lu-177 injection. If measurement results allow, patient will discharged from isolated rooms. [6-8]

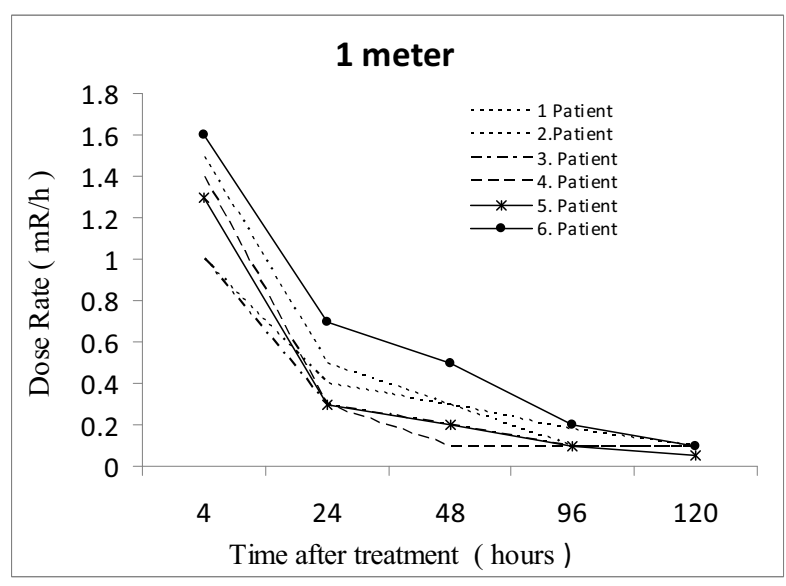

Figure 3. Dose rate measurements results at $1 \mathrm{~m}$.

\section{References}

1. J. Fitschen, et al., Z Med Phys. 21 (4) 266-73 (2011)

2. G.S. Limouris,Front Oncol. 28220 (2012)

3. S. Banerjee, et al., Nucl. Med. Biol., 31 753-759 (2004)

4. B.L.R. Kam, et al., Eur J Nucl Med Mol Imaging. 39 103-11 (2012)

5. S. Walrand, et al., Eur J Nucl Med Mol Imaging. 2011;38(Suppl 1):S57-S68. doi: 10.1007/s00259011-1771-7.

6. G.A. Kaltsas, D. Papadogias, P. Makras, A.B. Grossman, Endocr Relat Cancer. 12 683-99 (2005)

7. E.J. Rolleman, et al., Eur J Nucl Med Mol Imaging. 37(5):1018-1031 (2010)

8. N. Singh, et al., Indian J Nucl Med. 26(3): 135-138 (2011) 